\title{
X-ray Method for Structural Study of Liquid Cyclohexanol
}

\author{
(INDEX: 99-103/2010 Copernican Letters ${ }^{\circledR}$ Vol 1)
}

\author{
Krzysztof Nowakowski, Henryk Drozdowski \\ Faculty of Physics, A. Mickiewicz University, ul. Umultowska 85, \\ 61-614 Poznań, Poland \\ Email: riemann@amu.edu.pl
}

\begin{abstract}
An angular distribution of X-ray scattered intensity in liquid cyclohexanol was measured. The electron density radial distribution function was calculated. The structural parameters, the mean distances between the neighbouring molecules and the mean radii of coordination spheres were found. Mean most often recurring intermolecular distances, obtained from an analysis of the difference radial distribution function, are calculated as: $r_{1}=4.22 \AA, r_{2}=$ $5.10 \AA, r_{3}=8.22 \AA$.
\end{abstract}

\section{Introduction}

X-ray diffraction is a very efficient method of studying the structure of matter in both solid and liquid phase [1]. However, to the best of our knowledge, no reports have been published on the structure of cyclohexanol $\mathrm{C}_{6} \mathrm{H}_{11}-\mathrm{OH}$ in the crystalline state [2].

This paper reports the first studies of liquid cyclohexanol performed by the counter method for the range of the angular measurements intensity extended to the value of $2 \Theta=120^{\circ}$. Also for the first time the range of distances in the calculations of the differential radial distribution function (DRDF) for liquid cyclohexanol was extended to include the intermolecular distances of over $20 \AA$.

The aim of the study was to establish the role of the cyclohexane ring and the functional group $-\mathrm{OH}$ attached to it at the equatorial position in configurations of the molecules of the liquid studied. These results can be interpreted in terms of a simple model of local arrangement of molecules, which probably can be valid for a larger class of molecular liquids, i.e. polar monosubstituted derivatives of cyclohexane. Cyclohexanol samples of 99\% purity were purchased from AldrichChemie (Germany).

Cyclohexanol is used as a solvent and a substrate for production of synthetic fabric (nylon, perlon, stylon).

\section{Experimental method}

$\mathrm{X}$-ray scattering in liquid cyclohexanol $\mathrm{C}_{6} \mathrm{H}_{11}-\mathrm{OH}$ (melting point $23^{\circ} \mathrm{C}$, boiling point $\left.161^{\circ} \mathrm{C}\right)$, at a temperature of $(20.0 \pm 0.1)^{\circ} C$, was measured by applying MoKa radiation, $\quad \lambda=0.71069 \AA$, monochromatized by a graphite crystal. The scattered intensity distribution was measured by a goniometr HZG-3 for the angles $6^{\circ} \leq 2 \Theta \leq 120^{\circ}$ at every $0.2^{\circ}$, where $2 \Theta$ is the scattering angle.

The measurements were performed using the transmission technique [3]. The movement of the counter on the goniometer circle is coupled with the rotation of the table with the sample about the vertical axis of the goniometer at the ratio 2:1. The synchronization of the two movements means that the input slit of the counter is always at the point of intersection of the Rowland circle with goniometer circle (Fig. 1). The geometry of the system was verified by testing the agreement between the results measured towards increasing and decreasing angles. 
The scattered X-ray intensities were measured by a VA-G-120 proportional counter (made Otto Schön, Germany). The diffracted X-ray pulses are counted in the time of $40 \mathrm{~s}$. The deviation in intensity due to instability of the diffractometer work were of about $1 \%$ in the whole range considered.

The studies were carried out with a special cuvette with exchangeable plates and closed by mica windows of thickness $0.025 \pm 0.001$ $\mathrm{mm}$. The distance between the mica windows was $1.00 \pm 0.01 \mathrm{~mm}$.

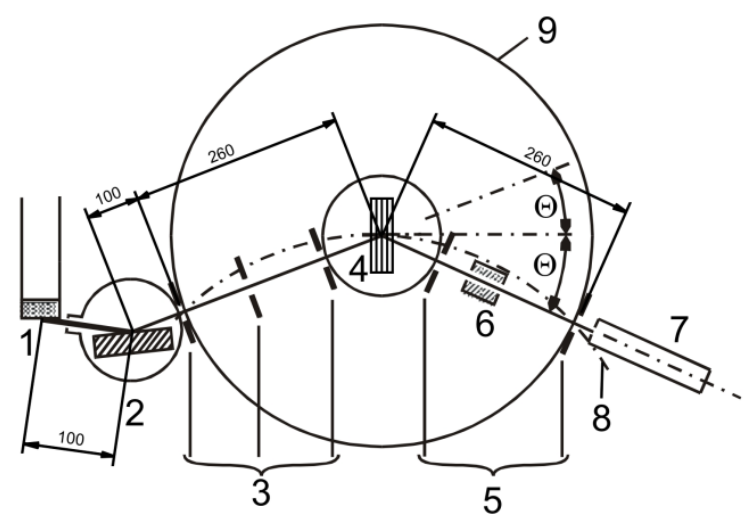

Fig.1. The focusing of the beam passing through the monochromator and goniometer with the preparation studied: 1 - X-ray lamp anode, 2 - monochromator, 3 - a system of input slits of the goniometer, 4 - cuvette with the liquid studied, 5 - a system of output slits, 6 - Soller slits, 7 - radiation counter, 8 - goniometer circle, 9 - Rowland circle; distances expressed in $\mathrm{mm}$

\section{Calculations}

The experimental function of angular distribution of the scattered X-ray intensity was corrected to include the polarization and absorption factors [4], and then normalized [5]. The intensity in the electronic units was presented as a function of $S$ defined by the formula $S=(4 \pi \sin \Theta / \lambda)$, where $\Theta$ is the Bragg angle, $\lambda$ is the wavelength of $X$-ray radiation [6].

The modified Warren-Krutter-Morningstar method was applied to obtain the DRDF. The method is described in details in $[7,8]$.
The calculation of the DRDF was performed with the use of the computer program LIQUIDS written by Drozdowski [9]. The DRDF was calculated by the Simpson integration method for $0 \leq \bar{r} \leq 20 \AA$ at every $0.05 \AA$, based on the parabolic interpolation.

\section{4. $\quad$ Results and discussion}

The normalized angular-distribution function $I(S)$ (Fig. 2) was found to have the main maximum for $S=1.43 \AA^{-1}$ which corresponds to the mean intermolecular distance in liquid cyclohexanol of $\bar{R}=(5.11 \pm 0.15) \AA$ determined directly from Voigtlaender-Tetzner equation [10]. Small-angle scattering results $\left(0^{\circ}<\Theta \leq 3^{\circ}\right)$ were extrapolated to the origin of the coordinate system using second-order function.

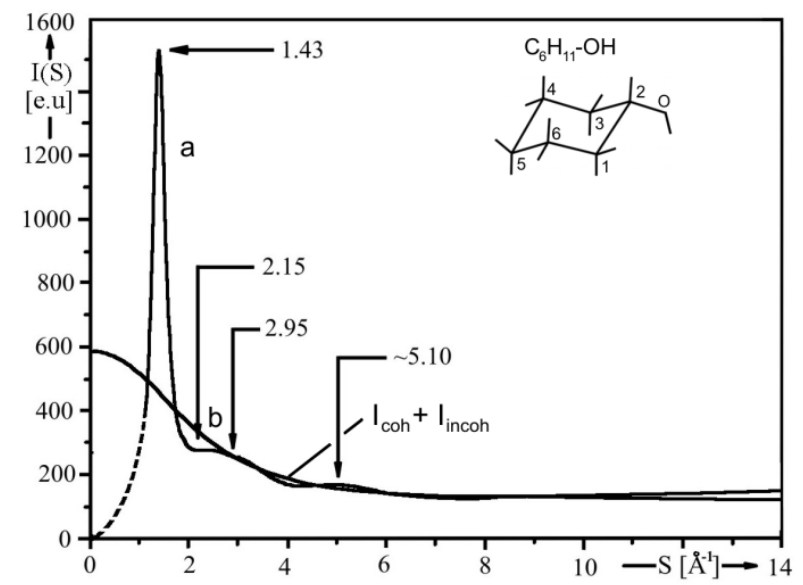

Fig.2. Normalized, experimental curve of angular distribution of X-ray scattered intensity (a), and total independent scattering curve (b) for cyclohexanol The theoretical function (Fig. 2b) was obtained as a sum of the intensities $I_{c o h}(S)$ of coherent scattering and $I_{\text {incoh }}(S)$ of incoherent scattering [11]. The position of the main maximum was read out with an accuracy $\Delta S= \pm 0.01 \AA^{-1}$. 
The application of Fourier transforms [12] to the experimental curve $I(S)$ gave the DRDF presented in Fig. 3. The shape of the DRDF provide information on the structure of liquid cyclohexanol. The positions of the maxima on the functions presented in Figs 2 and 3 made the basis for the most probable model of molecular arrangements in liquid cyclohexanol.

The maxima on the DRDF, Fig. 3, have been ascribed to the following pairs of atoms:

$C(1)-H=1.10 \AA, \quad C(1)-C(2)=1.54 \AA$,
$C(1) \cdots O H=2.56 \AA, \quad C(1) \cdots C(4)=2.90 \AA$,
$C(4) \cdots O H=3.60 \AA \quad(C \cdots C$ is the nonbonded carbon atoms).

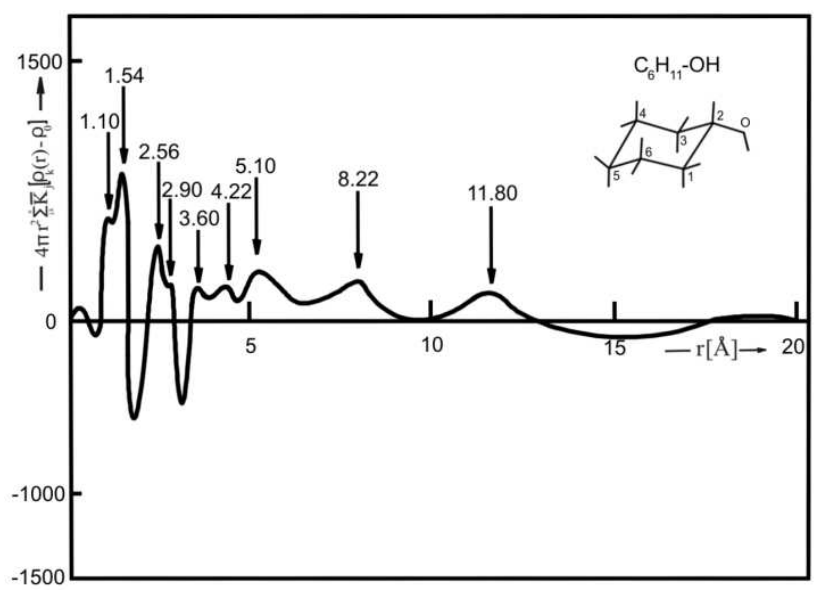

Fig.3. The differential radial distribution function for liquid cyclohexanol $\mathrm{C}_{6} \mathrm{H}_{11}-\mathrm{OH}$

From the known interatomic distances and valence angles, and assuming the van der Waals atomic radii [13] determined by $X$ ray methods for crystalline organic compounds, one can construct the cyclohexanol molecule and hence perform an analysis of the mutual dispositions of molecules in the liquid. The cyclohexanol molecule can be described by three values (length $x$ width $x$ height) which are obtained in orthogonal projections of the model onto three mutually perpendicular planes (Fig. 4). A molecule of cyclohexanol takes on the average a volume $\bar{V}=173.7 \AA^{3}$, whereas an increment of the molecule volume is $V^{i n c r}=105.7 \AA^{3}$. The specific volume of the molecule $V^{\text {incr }}$ was determined on the basis of the volume increments corresponding to the contribution of particular kinds of atoms in the total volume. The mean volume per one molecule $\bar{V}$ of the liquid was calculated from the known macroscopic density, molecular mass, and the Avogadro number [10]. The packing coefficient, defined by Kitaigorodsky [14] as a ratio of the specific volume of the molecule to the volume per a molecule in a given liquid, takes a value of 0.61 . This value falls within the range determined for the liquid phase - from 0.51 to 0.68 .
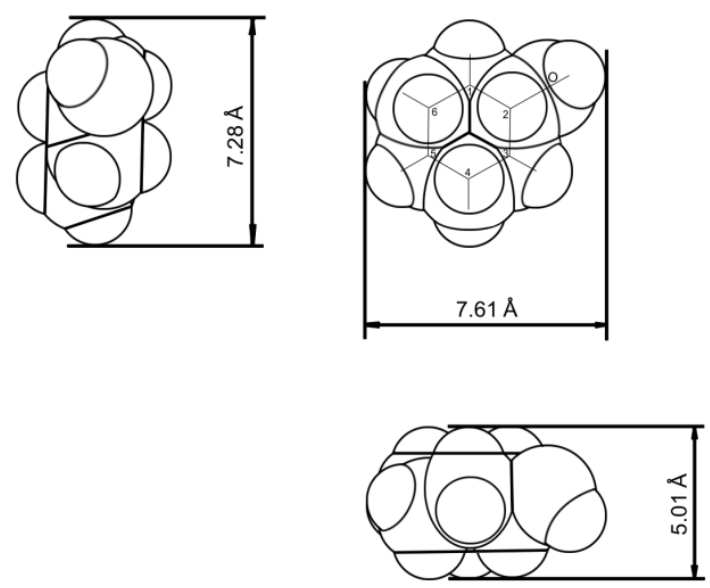

Fig.4. A model of cyclohexanol molecule $\mathrm{C}_{6} \mathrm{H}_{11}-\mathrm{OH}$ $\left(d=0.958 \mathrm{~g} / \mathrm{cm}^{3} ; M=100.2 \mathrm{~g} / \mathrm{mol}\right)$ projected onto three orthogonal planes

The distance between the centres of two neighbouring molecules is defined by van der Waals atomic radii [13]. The mean intermolecular distance for liquid studied $\bar{r}=(4.21 \pm 0.10) \AA$, Fig. 5, corresponds to the case of plane-parallel molecular arrangement. The attractive dipole interaction between neighbouring molecules can favour the plane arrangement of molecules one over another 
(i.e. the antiparallel one), making such a configuration relatively more stable.

The position of maximum at $\bar{r}=(11.80 \pm 0.15) \AA$ for cyclohexanol (Fig. 3) corresponds to the radii of the second coordination sphere [15].

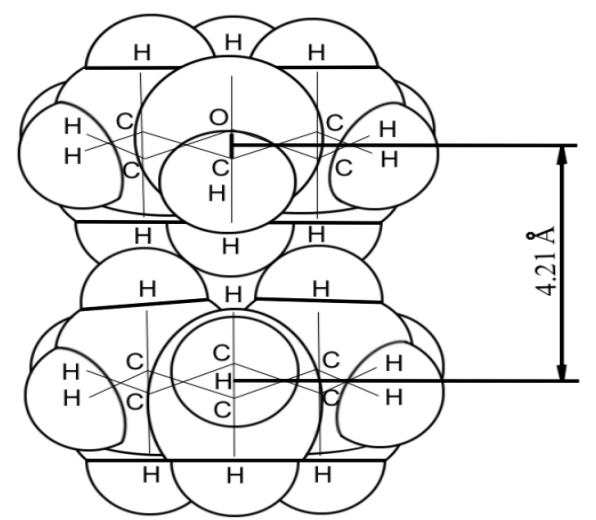

Fig.5. Plane-parallel packing of molecules in liquid cyclohexanol $\mathrm{C}_{6} \mathrm{H}_{11}-\mathrm{OH}$

In the liquid cyclohexanol the molecules are arranged with their cyclohexyl rings in parallel. Because of the permanent dipole moment of the molecule, cyclohexanol $\mu=1.85 \mathrm{D}$, the neighbouring molecules are arranged so that their dipolar moments are antiparallel. In liquid cyclohexanol only in the antiparallel conformation the distance between the centres of the hydroxyl group is $(\mathrm{OH})_{1} \cdots(\mathrm{OH})_{2}=(8.21 \pm 0.15) \AA$ (Fig. 6).

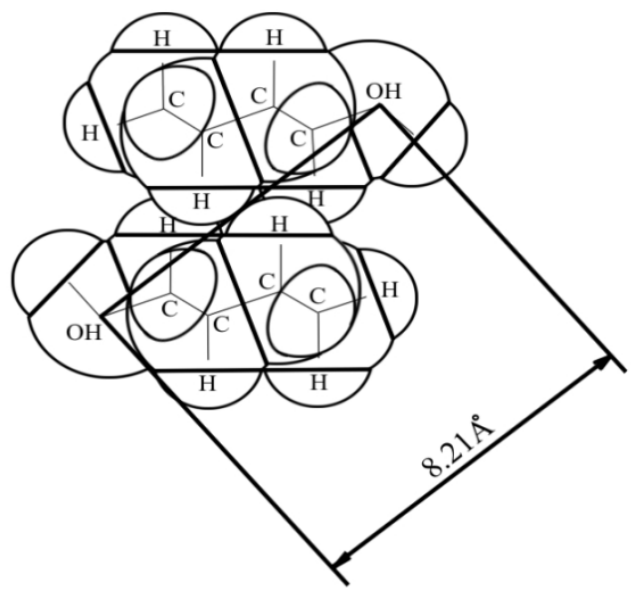

This distance corresponds to the position of the eight maximum on the DRDF (Fig. 3).
Fig.6. The antiparallel arrangement of molecules in liquid cyclohexanol $\mathrm{C}_{6} \mathrm{H}_{11}-\mathrm{OH}$

\section{Conclusions}

The atomic and molecular structure of cyclohexanol, $\mathrm{C}_{6} \mathrm{H}_{11}-\mathrm{OH}$ at $(20.0 \pm 0.1)^{\circ} \mathrm{C}$ was investigated using the $\mathrm{X}$-ray diffraction method.

The most important results of this study are listed below.

- New experimental data on the structure of cyclohexanol in liquid phase.

- New information on mutual arrangement, orientation, and packing of the molecule studied.

- Proposition of models of intermolecular binary radial correlations in the antiparallel orientation of dipole moments in the liquid studied.

- The presence of short-range ordering reaching about $20 \AA$ in the molecular liquid studied has been proved.

A molecule of liquid cyclohexanol, in the conditions of the present experiments, occupies on the average a volume of $\bar{V}=173.7 \AA^{3}$. Now, the calculated mean most often recurring least mutual distances between liquid cyclohexanol molecules, from our analysis of the differential radial distribution function, amount to $\bar{r}_{1}=(4.22 \pm 0.10) \AA, \quad \bar{r}_{2}=(5.10 \pm 0.15) \AA$ and $\bar{r}_{3}=(8.22 \pm 0.15) \AA$. From these data, and from an analysis of the possible models of molecular disposition and packing in the liquid, a most-probable spatial moleculecentre model of liquid cyclohexanol is constructed.

The data obtained from X-ray diffraction measurements regarding intermolecular distances, dispositions, orientations and packing in liquid cyclohexanol are in satisfactory agreement with the results of empirical and semi-empirical MO-LCAO calculations [16]. The results have also been confirmed by the conformational analysis based on minimization of the potential energy. 


\section{REFERENCES}

[1] H. Ohno, K. Igarashi, N. Umesaki, K. Furukawa, „XRay Diffraction Analysis of Ionic Liquids.", Trans Tech Publications. Switzerland-Germany-UK-USA, 1994.

[2] J. Robertson, „Organic Crystals and Molecules.”, Cornell University Press, Ithaca, New York, 1963; „Cambridge Structure Data Base (CSD).", 2010.

[3] D. North, C. Wagner, "An X-ray Transmission Diffractometer with a High Temperature Camera for the Study of Liquids.", Journal of Applied Cystallography 1969, s. 149-155, nr 2.

[4] P. Schwager, K. Bartels, R. Huber, "A Simple Empirical Absorption-Correction Method for X-ray Intensity Data Films.", Acta Cystallographica 1973, s. 291-295, nr A29.

[5] J. Krogh-Moe, "A Method for Converting Experimental X-ray Intensities to an Absolute Scale.", Acta Cystallographica 1956, s. 951-953, nr 9.

[6] H. Klug, L. Alexander, "X-Ray Diffraction Procedures for Polycrystalline and Amorphous Materials.", John Wiley, New York-London, 1974.

[7] H. Drozdowski, „Local structure and molecular correlations in liquid 1-methylnaphthalene at 293 K.", Chemical Physics Letters 2002, s. 53-60, nr 351.

[8] H. Drozdowski, "X-ray scattering in liquid $\beta$ naphthalene derivatives.", Journal of Molecular Structure 2001, s. 83-92, nr 595.

[9] H. Drozdowski, „X-ray Diffraction Study of Structure and Molecular Correlations in Liquid Naphthalene Derivatives.", Scientific Publishers Adam Mickiewicz University, Poznań, 2001 (Polish text with a summary in English).

[10] A. Mikusińska-Planner, „The application of X-ray investigation of liquid structure in determination of packing coefficient of molecules in liquid 2-methyl-2propanol.", Journal of Molecular Liquids 1986, s. 203-212, nr 31.

[11] H. Drozdowski, K. Nowakowski, „X-ray Diffraction Studies of Liquid Methylcyclohexane $\mathrm{C}_{6} \mathrm{H}_{11}-\mathrm{CH}_{3}$ Structure at 293 K.", Synchrotron Radiation in Natural Science 2007, s. 34-36, nr. 6

[12] R. Lovell, G. Mitchell, A. Windle, „An Improved Transformation Procedure for Radial Distribution Function Analysis.", Acta Crystallographica 1979, s. 598-603, nr A35.

[13] L. Pauling, P. Pauling, „Chemistry.”, W.H. Freeman and Company, San Francisco, 1985.

[14] A. Kitaigorodsky, „Molecular Crystals and Molecules.", Academic Press, New York and London, 1973.

[15] I. Hargittai, W. Orville-Thomas, „Diffraction Studies on Non-Crystalline Substances.", Akademiai Kiado, Budapest, 1991.

[16] J. Kriz, J. Jakes, „Conformational Structure of Ethylbenzene and its Correlations with Semiempirical MO-LCAO Calculations.", Journal of Molecular Structure 1971, s. 367-372, nr 12. 